\title{
Colorectal goblet cell sialomucin heterogeneity: its relation to malignant disease
}

\author{
K SUGIHARA, ${ }^{*} \dagger$ J R JASS,$* \ddagger$ \\ From the *Imperial Cancer Research Fund, Colorectal Cancer Unit, St Mark's Hospital, London, the \\ $\dagger$ Department of Surgery, University of Tokyo, Japan, and the $\ddagger$ Department of Histopathology, Medical College \\ of St Bartholomew's Hospital, London
}

SUMMARY Mucus secreted by colorectal cancer differs in three respects from that produced normally: an overall reduction, a loss of $\mathrm{O}$-acetyl substituents in sialic acid, and an increase in neutral mucin. Similar changes have been reported in apparently normal mucosa bordering colorectal cancer. "Normal" left sided colorectal mucosa from 32 patients with rectal cancer was studied. Each case was matched by age and sex to a patient with diverticular disease and a patient with irritable bowel syndrome. Twenty five patients with right sided cancer were matched to patients with Crohn's disease. Sections were stained with mild periodic acid Schiff (mPAS) (selectively stains N-acetyl sialic acid lacking in $\mathrm{O}$-acetyl group) and other closely related techniques. Reactions were graded negative, weak, and intense. An intense reaction was found in $9 \%$ of cases; there was no difference between the various matched groups. Phenylhydrazine interposition failed to block the mPAS effect, indicating that a positive result was due to a deficiency of sialic acid with $\mathrm{O}$-acetyl substituents rather than neutral mucin. Different staining patterns in left and right colon were probably due to differing ratios of total sialic acid:fucose. These findings indicate a hitherto unsuspected colorectal goblet cell sialomucin heterogeneity within the general population, but no association with neoplastic disease is apparent.

Colorectal goblet cells are believed to secrete mainly acid mucin but also small amounts of neutral mucin. ${ }^{1}$ There are important regional differences in the colon. The right colon secretes more neutral mucin ${ }^{2}$ (higher molar ratio of fucose:sialic acid) and expresses more (fucose rich) blood group antigens. Biochemical differences are found in sialic acid, with greater sensitivity to neuraminidase digestion being evidenced by saponified sialic acid in the left colon. ${ }^{2}$ The structural basis of this change is not known. Most sialic acid residues bear $\mathrm{O}$-acetyl groups in the polyhydroxy side chain or at position $\mathrm{C}_{4}$, or both.

Colorectal cancer usually secretes less mucus than normal mucosa, which hinders its detailed characterisation. Biochemical and histochemical studies, however, have shown important qualitative changes. These include an increase in the amount of neutral mucin and a reduction in the proportion of side chain and $\mathrm{C}_{4}$ substituted sialic acid. ${ }^{2}$ Modified periodic acid Schiff techniques, periodate borohydride/potassium hydroxide/periodic acid Schiff (PB/KOH/PAS) ${ }^{3}$ and periodate thionin Schiff/potassium hydroxide/ periodic acid Schiff (PT/KOH/PAS) ${ }^{4}$, were developed by Culling et al to show changes in sialic acid composition - that is, loss of O-acetyl substituents and increased expression of $\mathrm{N}$-acetyl sialic acid Unfortunately, neither of these methods distinguishes $\mathrm{N}$-acetyl sialic acid from neutral mucin; both are oxidised by periodic acid. This important lack of specificity has now been corrected, but earlier conclusions are open to question.

Several groups have reported "cancer-associated" changes in "normal" colorectal goblet cells adjacent to cancer. A combined biochemical and histochemical study has shown that the mucosa in the proximity of colorectal cancer differs from normal in secreting a reduced proportion of O-acetyl sialic acid. ${ }^{5}$ In $24 \%$ of cases no change was observed, but in $42 \%$ there was a focal reduction, and in $34 \%$ a moderate to severe field reduction was noted. The histochemical observations suggested that the findings were not due to a general loss of sialic acid but due to the deletion of O-acetyl substituents. ${ }^{5}$ Similar changes were reported by Katsuyama et al. ${ }^{6}$ Unfortunately, these studies did 1088 
Table 1 Interpretation of histochemical staining

\begin{tabular}{llll}
\hline & $\begin{array}{l}N \text {-acetyl sialic acid lacking in O-acetyl } \\
\text { groups }\end{array}$ & O-acetyl sialic acid & Neutral mucus \\
\hline mPAS & + & - & - \\
PAPS & + & + & - \\
KOH/PAPS & + & - & + \\
PAS & + & - & + \\
\hline
\end{tabular}

not include a control group. In contrast, Filipe's studies on sialic acid in "transitional mucosa" showed little change in $\mathrm{O}$-acetyl substitution. ${ }^{78}$ Two recent publications using control tissues indicated that sialic acid heterogeneity may be a feature of the general population. ${ }^{910}$ This further reinforces the need for caution in accepting the findings of Reid $e t a l^{5}$ and Katsuyama et al. ${ }^{6}$

This study was designed to clarify the current position by studying a large number of neoplastic and non-neoplastic colorectal specimens, using a simple battery of mucin histochemical techniques with an acceptable level of specificity.

\section{Material and methods}

Fifty seven specimens of colorectal cancer and adjacent mucosa were studied. Thirty two of the tumours were located in the rectum and 25 in the right colon. These groups were studied separately with their own controls to eliminate the influence of site on the pattern of mucus secretion. The mean age of patients with rectal cancer was 61.9 years (range 37-85). Mucosa immediately adjacent to each cancer was matched with uninflamed mucosa from a specimen resected from diverticular disease (by age and sex). A small fragment of mucosa removed $5 \mathrm{~cm}$ proximal to tumour was matched similarly with a normal rectal biopsy specimen from a patient with irritable bowel syndrome. The mean age of patients with right sided cancer was 49.8 years (range 30-70). Mucosa adjacent to each tumour was studied. This was a selected group of patients with a relatively low mean age, because appropriate controls are rarely obtained from middle aged to elderly patients in the absence of malignant disease. The control tissue was derived from right hemicolectomy specimens for ileal Crohn's disease and comprised histologically normal colonic mucosa. An additional 26 total colectomy specimens for chronic constipation were included in this study to increase the number of controls and, more importantly, to permit comparison of left and right sided mucosa from the same subject. In 23 of the cases mucosa was identified as originating from the right (ascending) colon or left (sigmoid) colon.

TECHNIQUES

All specimens were fixed in $10 \%$ neutral buffered for- malin, routinely embedded, serially sectioned at a thickness of $5 \mu \mathrm{m}$, and stained by haematoxylin and eosin and the following histochemical methods.

\section{A Mild sodium periodate oxidation and Schiff ( $m P A S)$}

This new technique was developed by Veh et al ${ }^{11}$ and is stated to be specific for $\mathrm{N}$-acetyl sialic acid lacking in O-acetyl groups.

1 Dewax and bring to distilled water.

2 Wash in $0 \cdot 1 \mathrm{M}$ acetate buffer, pH $5 \cdot 5$, at $2^{\circ} \mathrm{C}$ for five minutes.

3 Treat with $1 \mathrm{mM} \mathrm{NaIO}_{4}$ in $0 \cdot 1 \mathrm{M}$ acetate buffer, pH $5 \cdot 5$, at $2^{\circ} \mathrm{C}$ for 10 minutes.

4 Wash in $1 \%$ aqueous glycerol for five minutes.

5 Wash in distilled water for five minutes.

6 Treat with Schiff's reagent at room temperature for 15 minutes.

7 Wash three times in $0.5 \% \mathrm{~K}_{2} \mathrm{~S}_{2} \mathrm{O}_{5}$ in $0.05 \mathrm{M}$ hydrochloric acid for five minutes.

8 Wash in running tap water for five minutes.

9 Wash in distilled water for five minutes.

10 Dehydrate, clear, and mount.

\section{$B$ Periodic acid Schiff ${ }^{12}(P A S)$}

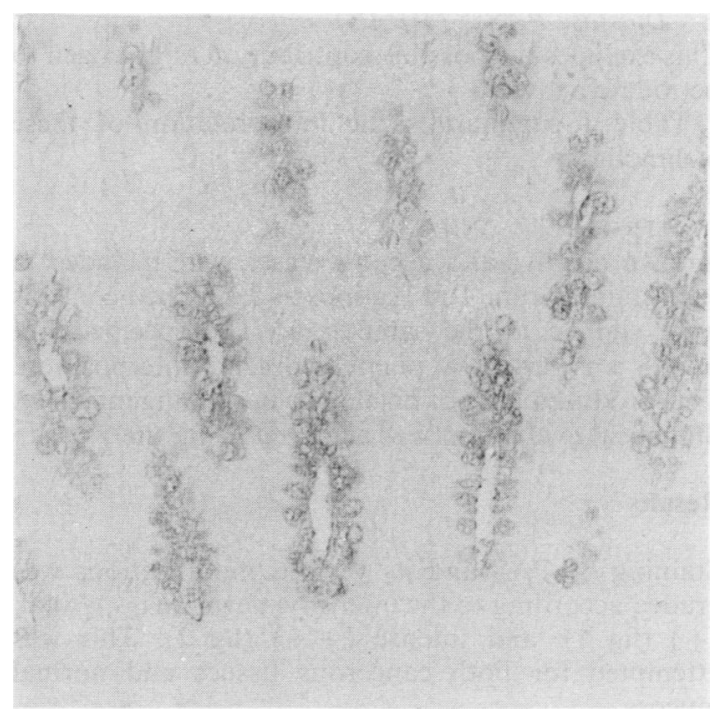

Fig 1 Diffuse weak positive staining with $m P A S . \times 75$. 


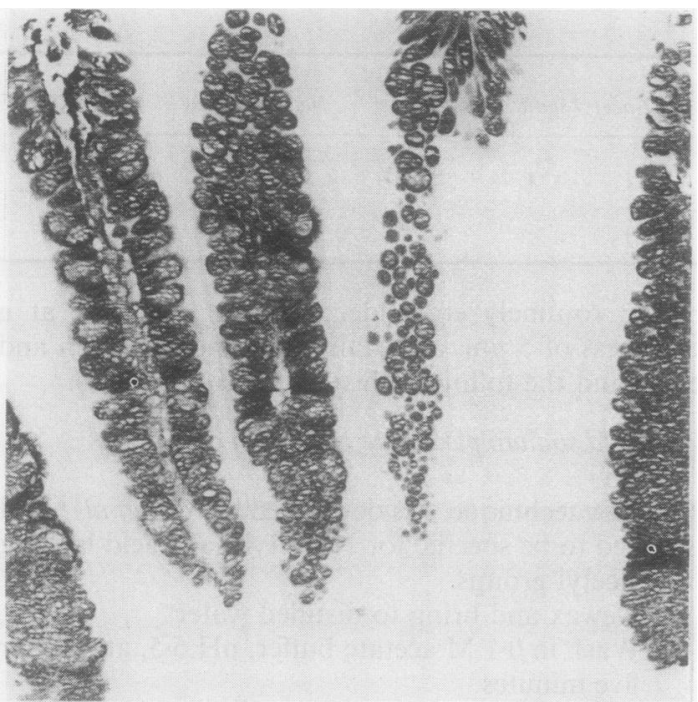

Fig 2 Diffuse intense positive staining with $m P A S . \times 75$.

C Periodic acid phenylhydrazine Schiff (PAPS)

This blocks periodate reactivity due to neutral mucin. ${ }^{13}$

\section{$D$ Potassium hydroxide periodic acid} phenylhydrazine Schiff (KOH/PAPS)

Ester on the polyhydroxy side chain is removed by saponification, ${ }^{14}$ which renders all types of sialic acid oxidisable by periodic acid, and neutral mucin is blocked with phenylhydrazine interposition.

\section{$E$ Diastase $P A S^{12}$ (DPAS)}

This excludes the possible contribution of glycogen to periodate activity.

Tabie 1 summarises the interpretation of these techniques.

\section{CONTROLS}

Known positive and negative cases were included in each staining run. Test sections and matched controls were stained at the same time. The specificity of mPAS was tested by phenylhydrazine interposition. This produced little or no change in staining intensity. Statistical evaluation was achieved using the $\chi^{2}$ test.

\section{Results}

Staining by PAS and its various modifications was graded according to the intensity: negative $(-)$; weak (+) (fig 1); and intense $(++)$ (fig 2). This was attempted for both cancerous tissues and normal mucosa.

(All mucosa, including that bordering cancer, are referred to as normal mucosa in the following.) Two patterns of mPAS staining were observed; a diffuse (graded,-+ , or ++ ) (figs 1 and 2 ) and a focal, in which small numbers of crypts were lined by intensely positive goblet cells (fig 3 ), whereas the background mucosa was negative or weakly stained. In any single crypt no differences were seen between goblet cells of crypt base, midcrypt, and surface epithelium.

\section{DIFFUSE MPAS STAINING IN NORMAL MUCOSA} FROM CANCER CASES AND CONTROL GROUPS There were no differences in the distribution of mPAS positive mucus between the three cancer matched control case studies (table 2). There were also no differences in mPAS staining between mucosa immediately adjacent to and $5 \mathrm{~cm}$ proximal to cancer of the rectum (table 2 ).

EFFECT OF PHENYLHYDRAZINE INTER POSITION UPON MPAS AND PAS STAINING, INCLUDING COMPARISON OF THE LEFT AND RIGHT COLON The effects of phenylhydrazine interposition were tested in normal mucosa adjacent to all carcinomas of the left and right colon. The effect on mPAS staining was either minimal or resulted in a very slight reduction only in intensity. This confirms the inability of mPAS to stain neutral sugars. On the other hand 8 PAS staining intensity was reduced by phenyl-0 hydrazine interposition (PAPS). In 16 of 32 cases of left sided mucosa there was a moderate reduction, and in 14 and seven of the 25 right sided samples the reduction was judged to be moderate and consid-

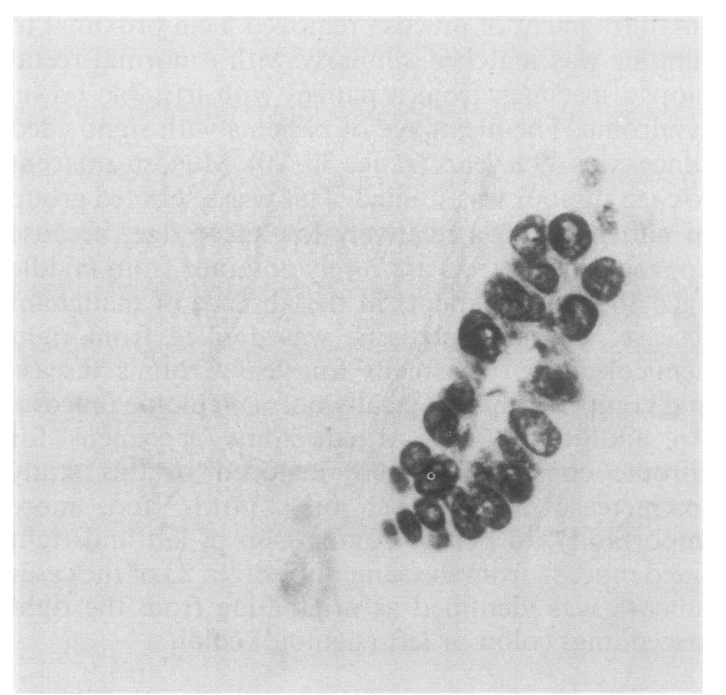

Fig 3 Focal mPAS positive crypt against negative background mucosa. $\times 75$. 
Table 2 Distribution of diffuse MPAS staining patterns in normal mucosa in three cancer matched control studies and series of colons removed for chronic constipation

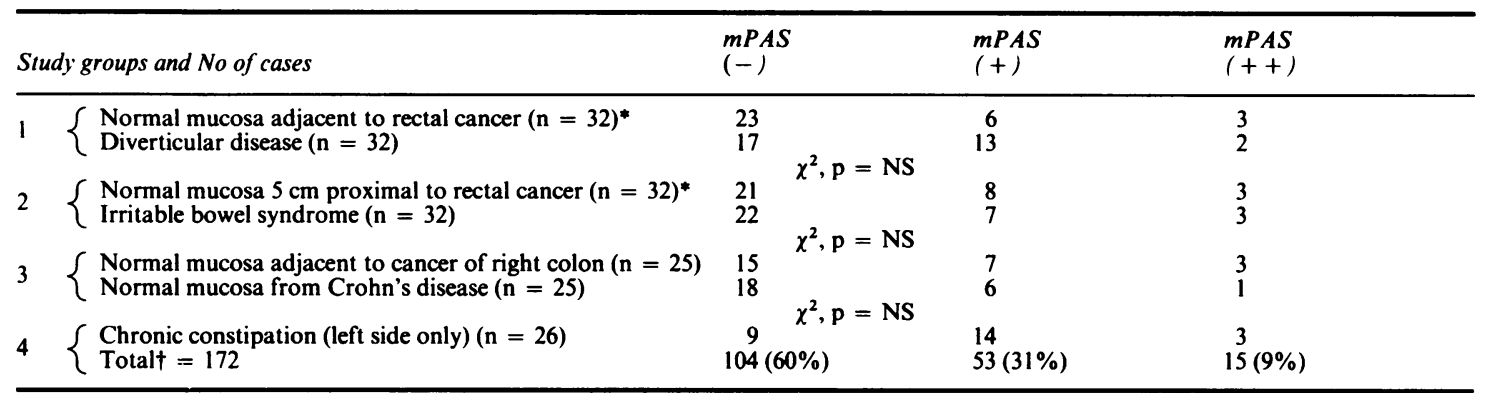

* Derived from same specimens.

†Excludes normal mucosa $5 \mathrm{~cm}$ proximal to rectal cancer.

erable, respectively. The differences between level of reduction in the left and right colon were significant $(p<0.01)$. These findings reflect the presence of neutral hexoses within goblet cell mucin of the large intestine and indicate that the right colon contains more neutral mucin than the left colon. This was confirmed in an intrapatient study of colectomy specimens removed for chronic constipation (table 3 ). In 17 of the 23 cases PAS blockade with phenylhydrazine interposition was judged to be greater in the right colon than in the left colon. In five cases no difference was detected, and in one case only the reduction in PAS staining seemed to be greater in the left side of the colon.

\section{EFFECT OF DIASTASE INTERPOSITION ON PAS STAINING}

This was performed on all normal mucosa adjacent to cancers of the left and right colon. Diastase interposition did not affect PAS reactivity, indicating a lack of epithelial glycogen stores.

\section{COMPARISON OF MPAS AND PAPS STAINING PATTERNS}

A total of 102 specimens were studied by PAPS as well as mPAS (table 4). In theory these techniques should have produced identical results. Both stain

Table 3 Intrapatient study of colectomy specimens removed for chronic constipation to show reduction of PAS staining following phenylhydrazine interposition in left and right colon

\begin{tabular}{|c|c|c|c|}
\hline $\begin{array}{l}\text { Reduction in left } \\
\text { colon }\end{array}$ & $\begin{array}{l}\text { No of } \\
\text { cases }\end{array}$ & $\begin{array}{l}\text { Reduction in right } \\
\text { colon }\end{array}$ & $\begin{array}{l}\text { No of } \\
\text { cases }\end{array}$ \\
\hline None to slight & $14=$ & $\begin{array}{l}\text { None to slight } \\
\text { Moderate } \\
\text { Pronounced }\end{array}$ & $\begin{array}{l}2 \\
8 \\
4\end{array}$ \\
\hline Moderate & $9 \leqq$ & $\begin{array}{l}\text { None to slight } \\
\text { Moderate } \\
\text { Pronounced }\end{array}$ & $\begin{array}{l}1 \\
3 \\
5\end{array}$ \\
\hline
\end{tabular}

oxidisable sialic acid (lacking O-acetyl substituents) and both fail to stain neutral mucin: with PAPS this is due to phenylhydrazine blockade; with mPAS the low concentration of periodic acid and low temperature combine to inhibit oxidation. For the intense and weak mPAS staining absolute concordance was, in fact, achieved (table 4). Just over one quarter of the mPAS negative cases, however, gave a weak result with PAPS (table 4). This could have been due to incomplete blockade of neutral mucin or increased sensitivity to oxidisable sialic acid.

MILD PAS AND KOH/PAPS STAINING-A COMPARISON OF LEFT AND RIGHT SIDED MUCOSA FROM COLECTOMY SPECIMENS FOR CHRONIC CONSTIPATION

With mPAS staining of the left and right colon, similar patterns were observed, although the intensity of staining was reduced in the right colon. In nine of 13 cases staining weakly in the left colon (table 2) little or no staining could be detected in the right colon. (In the fourteenth case it was not possible to identify a right sided sample.) Saponification with $\mathrm{KOH}$ renders all sialic acid oxidisable and therefore capable of giving a positive reaction with PAS. With phenylhydrazine interposition (neutral mucin blockade), this technique specifically shows most, if not all, forms of sialic acid. Although intense staining was achieved in most cases, in about half the intensity of staining was reduced in the right colon. Taken in conjunction with the findings for the effect of phenyl-

Table 4 Comparison of $m P A S$ and PAPS staining patterns

\begin{tabular}{|c|c|c|c|}
\hline$m P A S$ & $\begin{array}{l}\text { No of } \\
\text { cases }\end{array}$ & $P A P S$ & No of cases \\
\hline Negative & 62 & \multirow{2}{*}{$\begin{array}{l}\text { Negative } \\
\text { - Weak } \\
\text { - Weak } \\
\text { - Intense }\end{array}$} & \multirow{2}{*}{$\begin{array}{l}45 \\
17 \\
30 \\
10\end{array}$} \\
\hline $\begin{array}{l}\text { Weak } \\
\text { Intense }\end{array}$ & $\begin{array}{l}30= \\
10=\end{array}$ & & \\
\hline
\end{tabular}




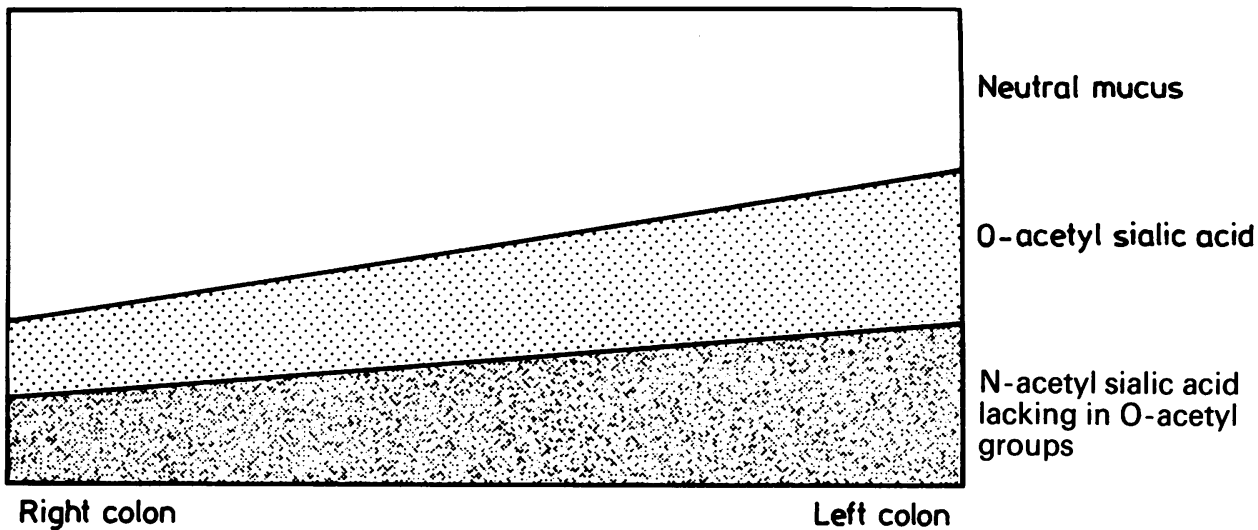

Fig 4 Schematic drawing to illustrate variation in amount of sialic acid and neutral mucin in normal colorectal mucosa. Staining intensities for $\mathrm{N}$-acetyl sialic acid lacking $\mathrm{O}$-acetyl groups (mPAS) and total sialic acid (KOH/PAPS) increase from right to left. There is no evidence from this study to suggest any intrapatient site related variation in ratio of $O$-acetyl: $N$-acetyl sialic acid.

hydrazine interposition it seems that an inverse relation exists between sialic acid and neutral sugars, with a higher total sialic acid:neutral sugar ratio existing in the left colon (fig 4). It also seems that the total amount of sialic acid is similar within a particular site of the colon in a series of subjects.

\section{MUCIN PROFILES IN CANCER $v$ ADJACENT NORMAL MUCOSA}

Difficulties were encountered because of considerable differences in the amount of mucus secreted by the cancers. Whereas six of 32 normal mucosal samples from the left colon gave a weak reaction with mPAS (table 2), 22 left sided cancers gave a weak reaction. This probably indicates an increased expression of Nacetyl sialic acid lacking in $\mathrm{O}$-acetyl groups within tumour mucus (presumably at the expense of $\mathrm{O}$-acetyl sialic acid). Three cancers gave an intense reaction with mPAS, but these were not the three cases that showed an intense positive reaction within normal adjacent mucosa.

In samples of cancer from the right colon six cases gave an intense reaction with mPAS. The adjacent normal mucosa was intensely positive in two, weakly positive in two, and negative in the remaining two cases. In the third case with an intensely mPAS positive normal mucosa the cancer mucus was negative. Cancers also showed increased PAS reactivity compared with that of adjacent normal mucosa. This effect was reduced by phenylhydrazine blockade in half the cases, showing that the increase was, in part, due to neutral mucin.

\section{FOCAL STAINING PATTERNS IN NORMAL} MUCOSA

In samples of normal mucosa giving a negative of weak diffuse result with mPAS one crypt, or some times small numbers of crypts (up to five), wers observed, in which the entire goblet cell population gave an intense positive result with mPAS. The reverse effect (focal negative or weak staining in an intensely positive background) was never seen. Focal positive crypts were also stained intensely with PAPS and PAS. These foci occurred more often in cancer cases than in controls when background staining was negative, but not when weak positive staining featured in the background (table 5).

\section{Discussion}

Mucosa adjacent to colorectal cancer differs from normal large bowel mucosa in giving a blue staining reaction with the high iron diamine alcian blue sequence. ${ }^{15}$ This method is based on differential ion binding and is not specific. ${ }^{16} \mathrm{~A}$ blue colour does not exclude the presence of sulphate, nor does a brown

Table 5 Focal positive MPAS staining against negative and weak positive background mucosa

\begin{tabular}{lcl}
\hline Background mucosa & Carcinoma & Control \\
\hline Negative & $11 / 38(28 \cdot 9 \%)^{*}$ & $3 / 35(8 \cdot 6 \%)^{*}$ \\
Weak positive & $4 / 13(30 \cdot 8 \%)$ & $4 / 19(21 \cdot 2 \%)$
\end{tabular}

No of focal positive cases/total.

${ }^{*} \chi^{2}$ test, $p<0.05$ 
reaction product necessarily signify the absence of sialomucin. Culling et al introduced a range of modified PAS techniques ${ }^{34}$ that are specific for $\mathrm{O}$ acetyl sialic acid. They showed that a loss of $\mathrm{O}$-acetyl substituents is found not only in benign and malignant neoplasms ${ }^{17}$ but is also evident in the mucosa bordering colorectal cancer. ${ }^{5}$ Their early studies suggested that a switch was taking place from $\mathrm{O}$-acetyl sialic acid to $\mathrm{N}$-acetyl sialic acid, but their original methods failed to distinguish between neutral mucin and $\mathrm{N}$-acetyl sialic acid. Although blockade of neutral mucin may be achieved by the interposition of phenylhydrazine ${ }^{1318}$ the complexity of their original methods has deterred many workers from investigating their utility as research or diagnostic tools.

In this study we used a battery of simple techniques to test the possibility that modifications in sialic acid might precede neoplastic transformation in the large intestine. The mPAS technique usually gave a negative result throughout the length of the colon. This supports the assertion by Veh et al ${ }^{11}$ that the method stains neither $\mathrm{O}$-acetyl sialic acid (except, possibly, 7 $\mathrm{O}$-acetyl sialic acid) nor neutral mucin; these are normally well represented within the colorectum. In the cases that were positive with mPAS phenylhydrazine interposition (for neutral mucin blockade) did not produce any appreciable reduction in the staining intensity. On the other hand, alcianophilia was reduced by prior digestion with neuraminidase (data not given). We are therefore confident that $\mathrm{N}$-acetyl sialic acid was detected by mPAS. Although weak and, less commonly, intense mPAS staining was observed in the mucosa bordering left and right sided cancers, these findings occurred with a similar incidence in control tissues from age and sex matched subjects. We therefore believe that constitutional factors may be responsible for goblet cell sialic acid heterogeneity within the general population.

Deficiency of $\mathrm{O}$-acetyl transferase activity, determined by homozygosity for a recessive gene, could account for the $9 \%$ of subjects secreting $\mathrm{N}$-acetyl sialic acid as opposed to $\mathrm{O}$-acetyl sialic acid. This would be analogous to the slow acetylator phenotype, which is caused by an $\mathrm{N}$-acetyl transferase defect. Most histopathologists must be familiar with the occasional rectal biopsy specimen showing an unusually intense result with routine PAS staining. Alternatively, some may have wondered at the weak staining of most rectal biopsy specimens. Differences in the PAS staining of rectal biopsy specimens do not reflect inconsistent laboratory practice but are determined by sialic acid heterogeneity within the general population. This heterogeneity is not associated with either blood group secretor state or acetylator phenotype (unpublished observations), nor is it related to neoplastic disease. This conclusion contradicts the findings of Reid et al, ${ }^{5}$ but receives support from Lev et al. ${ }^{9}$ It has been reinforced by our failure to show a concordance in staining patterns for cancer and adjacent mucosa.

Fig 5 shows the usual staining patterns in the left colon. mPAS and PAPS generally gave concordant results (table 4). PAPS, however, takes longer and entails use of the highly toxic chemical phenylhydrazine. When a weak or intense mPAS reaction
A
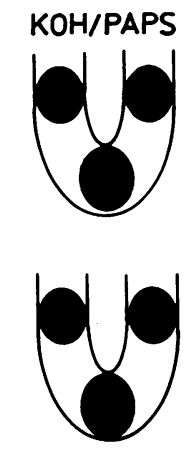

B
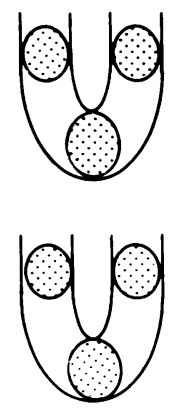
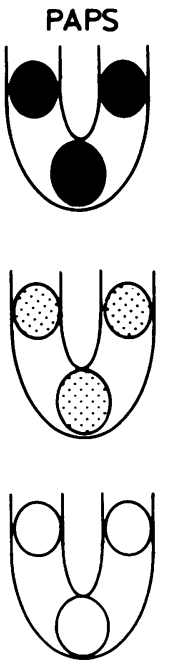

mPAS

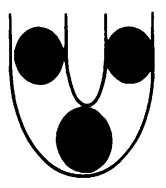

$\mathrm{N}$-acetyl sialic acid lacking in $\mathrm{O}$-acetyl groups predominant (least common)

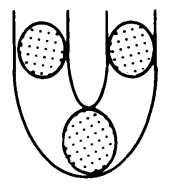

Mixtures of $\mathrm{N}$-acetyl and $\mathrm{O}$-acetyl sialic acid

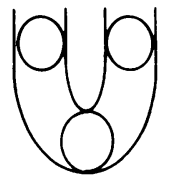

O-acetyl sialic acid predominant (most common)

Fig 5 Usual goblet cell staining patterns in left colon. (black $=$ intense, dots $=$ weak, white $=$ negative $)$. 

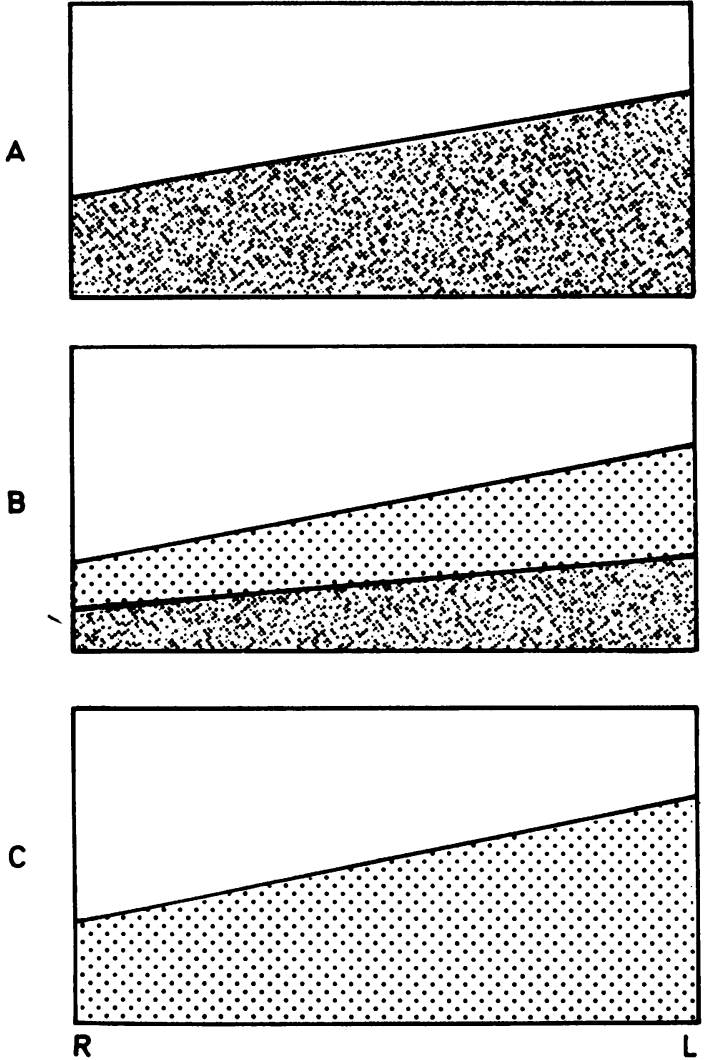

Fig 6 Schematic drawings to illustrate three main types of sialic acid expression based on patterns shown in fig $5 . \square=$ neutral mucus; $\square=O$-acetyl sialic acid; $\square=N$-acetyl sialic acid lacking in $\mathrm{O}$-acetyl groups.

occurred in the left colon of patients with chronic constipation, this was mirrored in the right colon, though the intensity was usually slightly diminished. $\mathrm{KOH} / \mathrm{PAPS}$ stains total sialic acid. In most of the cases tested the left and right colon again gave concordant results, though the intensity was relatively less strong in the right colon. On the other hand, the PAS method (which stains neutral sugars as well as some sialic acid moieties), resulted in more intense staining of the right colon. The principal terminal sugars in goblet cell mucin are probably fucose and sialic acid. ${ }^{19}$ Biochemical and histochemical studies $^{26}$ show that the fucose:total sialic acid ratio is higher in the right than the left colon. Our staining patterns support this view and have been illustrated schematically (fig 4). It is also relevant that Ulex europeus lectin (specific for fucose) binds selectively to the goblet cell mucin of the right colon ${ }^{20}$ and that right sided colonic mucus is known to express fucose rich blood group substances. ${ }^{21}$ We therefore suggest that the total amount of sialic acid depends on the region of colon under study, whereas the ratio of O-acetyl salic acid: $\mathrm{N}$-acetyl sialic acid is influenced by constitutional factors (fig 4). The ratio of $\mathrm{O}$-acetyl sialic acid and $\mathrm{N}$-acetyl sialic acid may be the same throughout the colon in any subject (fig 4). The relatively simple models depicted in fig 6 will be slightly complicated by the superimposition of focal changes shown by us and other workers. ${ }^{59}$

We found that focal mPAS positive crypts were more numerous in specimens harbouring cancer, but we interpret the change as a functional metaplasia rather than as a sign of early neoplastic change ${ }^{5}$ as identical changes are featured in metaplastic polyps of the colorectum. ${ }^{22}$ The existence of heterogeneity in large bowel sialic acid in the general population may have important implications. We have shown that mPAS positive sialic acid is susceptible to neuraminidase digestion (unpublished observations) and are now studying possible associations between the secretion of this variant of sialic acid and susceptibility to ulcerative colitis. Furthermore, it is now essential to establish the intrapatient sialic acid state when investigating changes associated with disease by histochemical or biochemical means.

\section{References}

1 Filipe MI. Mucins in the human gastrointestinal epithelium: review. Investigative and Cell Pathology 1979;2:195-216.

2 Reid PE, Culling CFA, Dunn WL, Ramey CW, Clay MG. Chems ical and histochemical studies of normal and diseased huma gastrointestinal tract. I. A comparison between histologicall normal colon, colonic tumours, ulcerative colitis and diverticular disease of the colon. Histochem $J$ 1984;16:235-51.

3 Reid PE, Culling CFA, Dunn WL. Saponification induced increase in the periodic acid-Schiff reaction in the gastrointestinal tract. Mechanism and distribution of the reactive substance. $J$ Histochem Cytochem 1973;21:473-82.

4 Culling CFA, Reid PE, Dunn WL. A new histochemical method for the identification and visualization of both side chain acylated and nonacylated sialic acid. $J$ Histochem Cytochem 1976;24:225-30.

5 Reid PE, Owen DA, Dunn WL, Ramey CW, Lazosky DA, Clay MG. Chemical and histochemical studies of normal and diseased human gastrointestinal tract. III. Changes of the epithelial grycoprotein in the mucosa close to colonic tumours. Histochem J 1985;17:171-81.

6 Katsuyama T, Ono K, Nakayama J, Akamatsu T, Honda T. Mucosubstance histochemistry of the normal mucosa and carcinoma of the large intestine. Galactose oxidase-Schiff reaction and lectin staining. Acta Pathol Jpn 1985;35:1409-25.

7 Greaves P, Filipe MI, Abbas S, Ormerod MG. Sialomucins and carcinoembryonic antigen in the evolution of colorectal cancer. Histopathology 1984;8:825-34.

8 Rogers CM, Cooke KB, Filipe MI. Sialic acids of human large bowel mucosa: $\mathrm{O}$-acylated variants in normal and malignant states. Gut 1978;19:587-92.

9 Lev R, Lance P, Camara P. Histochemical and morphologic studies of mucosa bordering rectosigmoid carcinomas: Comparisons with normal, diseased and malignant colonic epithelium. Hum Pathol 1985;16:151-61.

10 Muto T, Kamiya J, Sawada T, Agawa S, Morioka Y, Utsunomiya $\mathbf{J}$. Mucin abnormality of colonic mucosa in patients with familial polyposis coli. A new tool for early detection of 
the carrier? Dis Colon Rectum 1985;28:147-8.

11 Veh RW, Meessen D, Kuntz D, May B. In: Malt RA, Williamson RCN, eds. Colonic carcinogenesis. Lancaster: MTP Press Ltd, 1982:355-65.

12 Cook HC. Carbohydrates. In: Bancroft JD, Stevens A, eds. Theory and practice of histological techniques. 2 nd ed. London: Churchill Livingstone, 1982:180-216.

13 Spicer SS. The use of various cationic reagents in histochemical differentiation of mucopolysaccharides. Am J Clin Pathol 1961;5:393-407.

14 Culling CFA, Reid PE, Dunn WL. The effect of saponification upon certain histochemical reactions of the epithelial mucins of G.I. tract. J Histochem Cytochem 1971;19:654-62.

15 Filipe MI, Branfoot AC. Abnormal patterns of mucus secretion in apparently normal mucosa of large intestine with carcinoma. Cancer 1974;34:282-90.

16 McFadden DE, Owen DA, Reid PE, Jones EA. The histochemical assessment of sulphated and nonsulphated sialomucin in intestinal epithelium. Histopathology 1985;9:1129-32.

17 Culling CFA, Reid PE, Worth AJ, Dunn WL. A new histochemical technique of use in interpretation and diagnosis of adenocarcinomas and villous lesions in the large intestine. $J$ Clin Pathol 1977;30:1056-62.
18 Reid PE, Dunn WL, Ramey CW, Coret E, Trueman L, Clay MG. Histochemical studies of the mechanism of the periodic acidphenylhydrazine-Schiff (PAPS) procedure. Histochem $J$ 1984;16:614-9.

19 Hounsell EF, Feizi T. Gastrointestinal mucins. Structures and antigenicities of their carbohydrate chains in health and disease. Med Biol 1982;60:227-36.

20 Yonezawa S, Nakamura T, Tanaka S, Sato E. Glycoconjugate with Ulex europaeus agglutinin-I-binding site in normal mucosa, adenoma, and carcinoma of the human large bowel. JNCI 1982;69:777-85.

21 Denk H, Tappeiner G, Holzner JH. Blood group substance (BGS) as carcinofetal antigens in carcinomas of the distal colon. Eur J Cancer Clin Oncol 1974;10:487-90.

22 Jass JR, Filipe MI, Abbas S, Falcon CAJ, Wilson Y, Lovell D. A morphological and histochemical study of metaplastic polyps of the colorectum. Cancer 1984;53:510-5.

Requests for reprints to: Dr J R Jass, St Mark's Hospital, City Road, London ECIV 2PS, England. 